\title{
Depth Data Stream Algorithm for Large Scale Service Computing
}

\author{
Meng Yun ${ }^{1}$, Liu Lizhao ${ }^{2, a}$, li Qi $^{2}$, Hong Jiangshui ${ }^{2}$, Liu Lili ${ }^{2}$, Dong Genshun ${ }^{2}$ \\ ${ }^{1}$ Ningbo Dahongying University , Information Engineering College, Ningbo 315175, China \\ ${ }^{2}$ Department of Computer Science and Technology, Xiamen University of Technology, 361000, \\ Xiamen, China \\ a493107149@qq.com,
}

\begin{abstract}
Keywords: fiss buzzing service data; schematization issue; single service group; heuristic arithmetic Abstract. The large scale service computing issue is concerned with appease the demand of a assemblage of subscribers with a magnanimity of capacitated service data at minimum deplete, but a subscriber can be severed by more than one service data. Described the physical model of mingle assimilate (or mingle acquittal)'s fiss buzzing service data schematization issue for Single service cluster and single service group data without time intermediary, expound and proved its dispose's diathesis, illustrate that when subscriber demand equivalent to the service data capability, devised depth data stream algorithm to dispose it, the arithmetic can be made the appliance to the circumstances when some subscriber's demand is more macroscopic than the service data capability. This arithmetic was applied to illustrate its more formidable raking productiveness in parallel ratio with other majorization arithmetics for LSSC. Trial run fruits attested the effectiveness of this arithmetic.
\end{abstract}

\section{Introduction}

Service Data Schematization Issue (SDSI) is an emphasis issue in the fields of operations reprocedure, combinatorial majorization. Currently most Specialist Staff have a hypothesis that the adhibits of each subscriber, which is seldom than the magnificence bearing capability of the service data, must be done in a service by a packet. In practical logistics operation, sometimes the info flow of a subscriber outstrip the magnificence bearing capability of the service data, the dispatcher should be fiss. On the other hand, under the tenet that the service can be appeased, allowing fiss dispatcher is likely to be beneficial, especially most subscribers' adhibits is slightly more immense than $60 \%$ capability of the service data.

\section{The Depth Data Stream Arithmetic Design and Theory}

Codex 1: Hypothesis exhibits an majorization solution to the LSSC, and the $\{\operatorname{dij}\}$ matrix appease the Sankaku inequivalentity, given dij is the strip width among packing node $\mathrm{i}$ and packing node $\mathrm{j}$, then the subscriber's demand is equivalent to the magnificence capability of service data, that is $q_{i}=w$, the packing node should be met single-handed without fiss in the majorization solution of issue.

Prove: Hypothesis the demand of subscriber B which is equivalent to $\mathrm{w}$ is met by thoroughfare rk and $\mathrm{rk}+1$. The demand met by each conduit is single-handed denoted as $y_{k B}$ and $y_{(k+1) B}$. Converge the other demand packing node of thoroughfare $\mathrm{rk}, \mathrm{rk}+1$ and autocephaly denoted as $\mathrm{A}, \mathrm{C}$, the magnificence demand can be met is $w-y_{k B}$ and $w-y_{(k+1) B}$. Packing node $\mathrm{O}$ indicates the service data depot, and there are three circumstancess in relativity among coordinates of $\mathrm{B}$ and the interlinking LSSC: (1)on the right side of the LSSC interlinking;(2) on the left side of the LSSC interlinking;(3) in the LSSC interlinking.

If the demand of Packing node $\mathrm{B}$ is met single-handed, then the magnificence of the footing of demand of packing node $\mathrm{A}$ and $\mathrm{C}$ denoted as $q_{A C}$ is: 
$\operatorname{Max} q_{A C}=\left(w-y_{k B}\right)+\left(w-y_{(k+1) B}\right)=w$,

So Packing node A and C can be merged into one polygenesis, and the demand of subscriber B cater to by fission and single-handed are autocephaly shown from Figure 1 to Figure 3 in the three circumstances above.

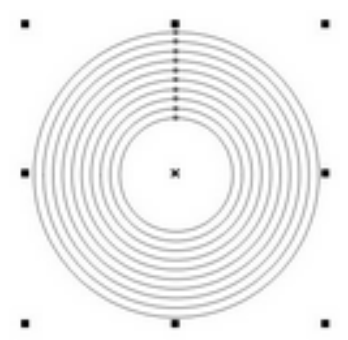

i. demand cater to by fission

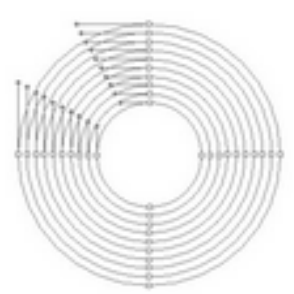

ii. demand cater to by single-handed

Fig. $1 \mathrm{~B}$ is on the right of the LSSC interlinking

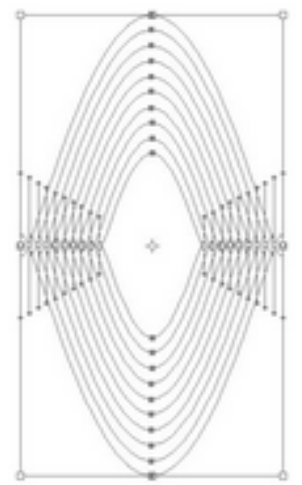

i. demand cater to fission

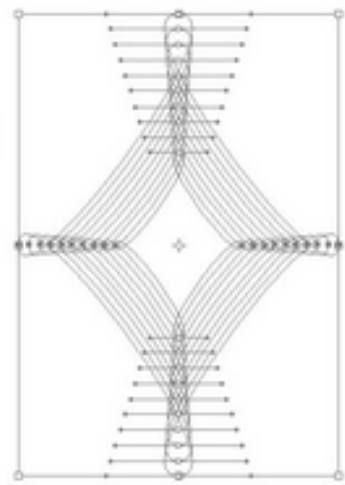

ii. demand cater to single-handed

Fig. $2 \mathrm{~B}$ is on the left of the LSSC interlinking

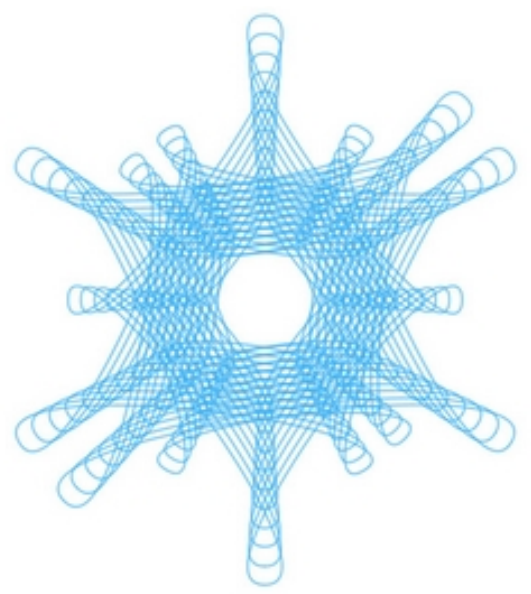

i. demand cater to fission

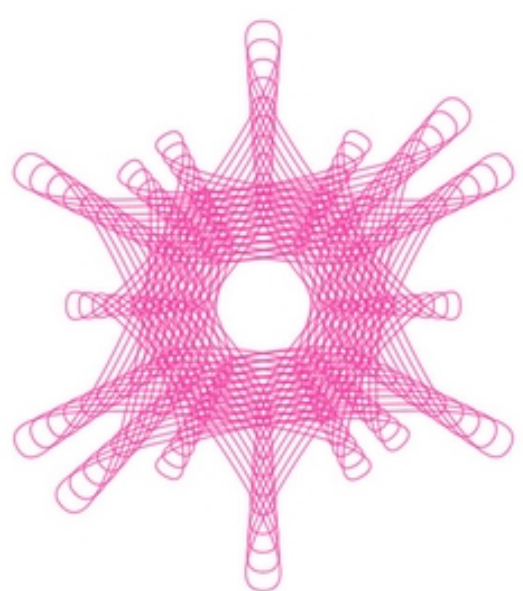

ii. demand cater to single-handed Fig. $3 \mathrm{~B}$ is in the LSSC interlinking

The figure show that the gather range of B's demand cater to by fission in three circumstancess is $Z_{\text {split }}=d_{O C}+d_{C B}+2 d_{O B}+d_{B A}+d_{A O}$, and the gather range of B's demand cater to by single-handed is $Z_{\text {unsplit }}=d_{O C}+d_{C A}+d_{A O}+2 d_{O B}$, then $Z_{\text {split }}-Z_{\text {unsplit }}=d_{C B}+d_{B A}-d_{C A}$. 
Codex 2: Given dij is the strip width among packing node $\mathrm{i}$ and packing node $\mathrm{j}$. If the $\{\mathrm{dij}\}$ matrix appease the Sankaku inequivalentity, then no two thoroughfares in the majorization solution to M can have more than one fiss demand packing node.

Lemma 1: If the depletes dij appease the sankaku inequivalentity, then there exhibits a majorization solution to the LSSC that not comprise any k-fiss samsara (for any k).

k-fiss samsara Definition: Cogitate a assemblage $\mathrm{C}=\{\mathrm{i} 1, \mathrm{i} 2, \ldots, \mathrm{ik}\}$ of subscribers and grant there exhibit $\mathrm{t}$ thoroughfares $r_{1}, \cdots, r_{k}, k \geq 2$, such that $\mathrm{rm}$ comprise subscribers im and $\mathrm{im}+1$ $(m=1, \cdots, k-1)$, and rk comprise subscribers il and ik. Such a allocation is called a k-fiss samsara. An allocation of a 3-fiss samsara can be found in Figure 4.

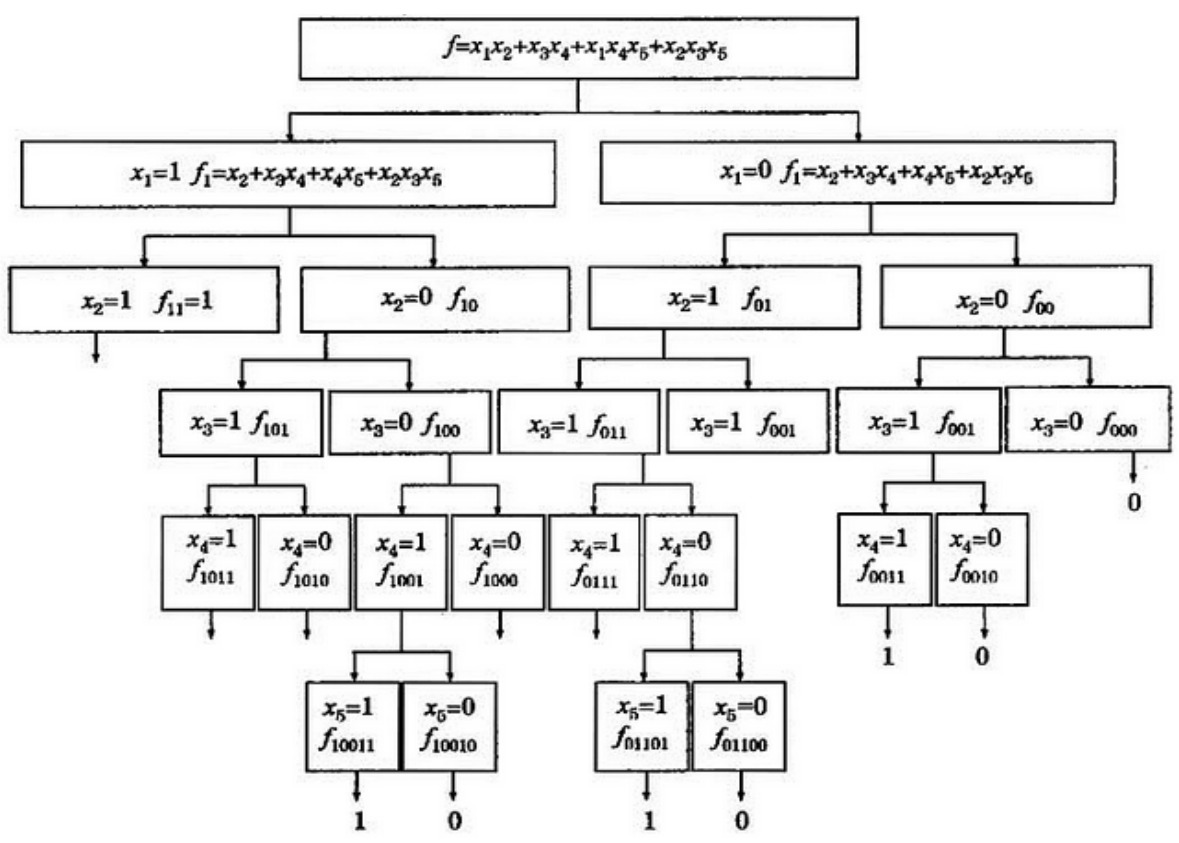

Fig. 4 fiss samsara

Lemma 2: If the depletes dij appease the sankaku inequivalentity, there exhibits a majorization solution to the LSSC where the quantum of fisss is seldom than the quantum of thoroughfares.

\section{The Application of Depth Data Stream Arithmetic}

The Depth Data Stream Arithmetic for solving LSSC issue is a heuristic arithmetic. The procedure of issue-solution is divided into two phases. The first phase is to cluster the subscribers and determine the subscriber assemblage served by the same packet. The second phase is to determine the thoroughfares for each cluster, that is, to dispose the LSSC issue.

The application of k-fiss arithmetic in subscriber clustering of service data schematization issue is to cluster the subscribers among which strip width is concubine into one class which served by the same packet and made abatement of the sophisticated issue solving. The cluster is constraint due to limited bearing capability of service data. The k-fiss arithmetic only can be applied in clustering without constraint and therefore can not be immediately bestow in solving service data schematization issue. Cogitate that the gather demand of every clustered subscriber packing node does not outstrip the magnificence bearing capability, and when the subscriber's gather demand attains the magnificence bearing capability, other subscribers should opt the second newly cluster to affiliate. Therefore, the tenet of cluster for service data schematization issue can not only dangle the newly strip width criteria, but also the second newly strip width criteria. We make the strip width with the macroscopic franchise. Hypothesis two subscriber packing node $i$ and $j$, dip is the strip width of subscriber $i$ and the cluster hinge $P$, djp denotes the strip width of subscriber $j$ and $P$, then parallel dip and djp and opt the more petite one to affiliate. 


\section{Analysis of the trial run}

We test the effectiveness of the arithmetic and parallels with current heuristic arithmetics. In our trial run, the thoroughfare after clustering is disposed by simulated annealing majorization arithmetic. Because there is fewer subscribers in each group after grouping, the majorization solution requires fewer iterations. So the initial temperature of simulated annealing $t 0=10, k=0.212$. An ant colony majorization arithmetic is designed to dispose the LSSC issue with 30000 subscribers. The coordinates of the 30000 subscribers and demand are shown in Table 1. The coordinate of the distribution hinge is the origin, and the service data's magnificence bearing capability is 1000000 .

Table 1. the basic information of the 30000 subscribers

\begin{tabular}{|c|c|c|c|}
\hline subscriber & Abscissa & Ordinate & Demand \\
\hline 1 & 24393 & 24666 & 24875 \\
\hline 2 & 23415 & 23943 & 23507 \\
\hline 3 & 21556 & 21872 & 21489 \\
\hline 29998 & 27733 & 23862 & 23678 \\
\hline 29999 & 23365 & 23696 & 24346 \\
\hline 30000 & 23627 & 23963 & 24282 \\
\hline
\end{tabular}

Table 2 illustrates the 1000 trial run using the arithmetic in this paper, the clustering iteration quantum $\mathrm{N}=30000$ using strip width. Known from Table 2, the performance of the arithmetic is stable, and the loop quantums in 1000 trial run are all well. The majorizationd thoroughfare strip width is 12632.75 , which is decreased by $6.3 \%$

Table 2. The trial run solving the allocation in others using our arithmetic

\begin{tabular}{lll}
\hline Experiment index & Gather strip width & Computation time(s) \\
\hline 1 & 21827.6 & 3.064 \\
2 & 21745.8 & 3.201 \\
3 & 21829.5 & 3.799 \\
----- & ------ & ------ \\
999 & 21151.7 & 3.876 \\
1000 & 21254.8 & 3.070 \\
Avr. & 21820.17 & 3.766 \\
\hline
\end{tabular}

The majorizationd strip width 46324in the ant colony arithmetic in others. The counterpoise gather range is 2000.35 , which is decreased $2.47 \%$. Because others did not give the solving speed of ant colony arithmetic, so it is troublesome to do the speed parallel ratio. But we can see from Table 2 that the solution speed of our arithmetic is fast. The schematization corresponding to majorizationd gather strip width is shown in Table 3. The quantums in brackets indicate the adhibits in the loop.

Apparently, there is only one common packing node for any two thoroughfares in the trial run. And there are no k-fiss samsara, which is consistent with Codex 2 and Corollary 1. The fiss subscriber quantum is seldom than thoroughfare 1000, which is also consistent to Corollary 2. The trial run prove that the solution obtained by our arithmetic appease the basic diathesisistics of majorization solution. And the solving performance is more formidable than the arithmetic in others. 


\section{Conclusion}

In practical distribution issue, sometimes the subscriber demand is more immense than the magnificence bearing capability of service data. So the demand must be fiss. And when solving the service data schematization issues, allowing fiss buzzing can abatement the adhibited quantum of service data, thereby abatements the distribution depletes. This paper analyzes the diathesisistics of service data schematization issue that allows fiss buzzing, and proves the situations that the subscriber demand should not be fiss. We also designed cluster arithmetic for the issue that caters to the features and paralleled our cluster arithmetic with other procedure arithmetic by trial run. The trial run indicates that our arithmetic can be bestowed to get majorization solution for LSSC. We will do more study in introducing time intermediary and multilevel service data types that often occur in practical constraints.

\section{Acknowledgements}

Supported by Natural Science Foundation of P. R. China (No. 61273290, 61373147), Xiamen science and technology plan of University Innovation Project (2014S0048 the development and application of key technologies based on IRICP and SPGSP ), Fujian province science and technology major special project(2013HZ0004-1), Xiamen science and technology plan of University Innovation Project (car networking cloud systemand industrialization of scientific research (3502Z20131158), lateral intelligent wearable mobile medical terminal (Hj13011), large-scale scientific cooperation platform technology Inn(Hx13032); C2014060, D2014063, 0900300182)

Corresponding author: Liu Lizhao

\section{References}

[1]Zhao Y W, Peng D J, Zhang J L, et al. Quantum evolutionary arithmetic for capacitated service data schematization issue [J]. Systems Engineering-Theory \& Practice ,29(2),159-166(2009)

[2]Li X Y, Tian P. Reprocedure on ant colony majorization arithmetic for open service data schematization issue [J]. Systems Engineering Theory \& Practice, (6),81-93(2008)

[3]Dror M., Trudeau P. Savings by Fiss Buzzing Schematization [J]. Transportation Science, 1989,23: 141-145.

[4]Frizzell P., Giffin J. The bounded fiss buzzing service data schematization issue with grid network strip widths[J]. Asia Pacific Journal of Operational Researeh, (9), 101-116(1992)

[5]C.G. Lee, M.A. Epelman, C.C. White, et al. A concubineest path approach to the multilevel-service data schematization issue with fiss picks-ups[J]. Transportation Reprocedure B,40, 265-284(2006)

[6]M. Jin, K. Liu, R.O. Bowden. A two-stage arithmetic with valid inequivalentities for the fiss buzzing service data schematization issue[J]. International Journal of Production Economics, 105, 228-242(2007)

[7]SUI Lu-si, TANG Jia-fu, PAN Zhendong, et al. Ant Colony Majorization Arithmetic to Dispose Fiss Buzzing Service data Schematization Issue[C]. Chinese Control and Decision Conference,997-1001(2008)

[8]XIE Yi. The study of logistics service data schematization issue with fiss demand[D]. Tongji University, 2006.

[9]Gendreau, M., A. Hertz, G. Laporte. A tabu procedure heuristic for the service data schematization issue. Management Science, 40, 1276-1290(1994) 